\title{
The Role of Modality in Notification Performance
}

\author{
David Warnock, Marilyn McGee-Lennon, and Stephen Brewster \\ Glasgow Interactive Systems Group, Department of Computing Science \\ University of Glasgow, Glasgow, G12 8QQ, UK \\ \{warnockd, mcgeemr, stephen\}@dcs.gla.ac.uk \\ http://MultiMemoHome.org
}

\begin{abstract}
The primary users of home care technology often have significant sensory impairments. Multimodal interaction can make home care technology more accessible and appropriate, yet most research in the field of multimodal notifications is not aimed at the home but at office or high-pressure environments. This paper presents an experiment that compared the disruptiveness and effectiveness of visual, auditory, tactile and olfactory notifications. The results showed that disruption in the primary task was the same regardless of the notification modality. It was also found that differences in notification effectiveness were due to the inherent traits of a modality, e.g.olfactory notifications were slowest to deliver. The results of this experiment allow researchers and developers to capitalize on the different properties of multimodal techniques, with significant implications for home care technology and technology targeted at users with sensory impairments.
\end{abstract}

Keywords: Multimodal interfaces, accessibility and usability, technology in healthcare.

\section{Introduction}

With an ageing population throughout Europe and similar trends emerging in other countries 25] it is apparent that preparations need to be made to support a larger aged population. While the post-war baby-boom is often cited as the primary reason for the predicted surge in people over retirement age, modern medicine has also increased life expectancy, suggesting that this trend will continue. As the population ages many will develop health problems that will require care, which is usually provided by family or paid carers. Increased demand for care services will make care at home uneconomical, which could lead to people being moved from their own homes into dedicated care homes where the quality of life is often perceived to be lower [18.

Home care technology can prevent this by helping care recipients to look after themselves, reducing their dependence on carers. This will enable them to continue living in their own homes with dignity and independence. A home reminder system is one type of home care system which can deliver alarms, warnings and information to the user. There are many applications for such reminders in the home: personal care reminders might remind the user to take

P. Campos et al. (Eds.): INTERACT 2011, Part II, LNCS 6947, pp. 572 588, 2011.

(C) Springer-Verlag Berlin Heidelberg 2011 
medication or eat dinner; lifestyle reminders could remind users about events and appointments; and home management reminders might remind users to lock doors and windows at night.

Users of home care technology are very likely to have one or more sensory impairments such as sight or hearing loss [12. Multimodal interaction uses one or more of the senses in order to convey information; as such it is ideal for creating more accessible systems for people with sensory impairments. Multimodal systems have the potential to allow interaction in a more appropriate way by altering the interaction modality based on factors such as user activity, social context, sensory impairment, preference, message complexity, message urgency and message sensitivity. For example, a potentially embarrassing toileting reminder might normally be delivered as a speech message, but could instead be delivered by private tactile notifications in a social setting.

In order for the designers of home care systems to use a range of modalities in this manner they need to know about the relative properties, capabilities and shortcomings of a variety of multimodal technologies. This paper empirically investigates the properties and effects of notifications delivered through different modalities in order to inform the design of multimodal technology for the home.

\section{Related Work}

Notifications can provide valuable information, aid multitasking, deliver timesensitive data and alert the user to important events. They can also be disruptive and irritating. McBryan \& Gray [21] describe a hypothetical scenario where notifications are delivered to a user, Fred, via his mobile phone. The mobile phone notifications irritate Fred when he is at home, so the notification system re-routes messages to be delivered via his television. Context sensitivity alongside a choice of modalities have stopped Fred from disabling his phone to stop the notifications. If the phone is legitimately unavailable, or Fred is not responding to the phone notifications, important messages can still be delivered using an alternative modality.

Many researchers have advocated using a range of modalities to provide more appropriate and acceptable interactions [1|2|16 19|21|22 28]. However, no guidelines or models exist that will help developers to include them, and most research in this area is directed towards the office or high-pressure environments such as aeroplane cockpits. In order to develop such guidelines, research is needed that will reveal how disruptive certain modalities are along with their general effectiveness as notifications.

A multimodal reminder system should not be disruptive in the home environment. If a notification interrupts the user it could create stress [1120], generate annoyance and anxiety [3], cause the user to make mistakes in the interrupted activity [17, alter the perceived difficulty of a task [3] and cause a person to speed up their activities [810 20]. Monk et al. 24] found that interruptions as short as 0.25 of a second were just as disruptive as interruption 5 seconds long. Berg et al. [5] investigated the cause of slips, trips and falls in older adults and found that most were considered 'avoidable' and blamed on 'hurrying too much'. Home care developers must be aware of these adverse affects in order to minimise their impact. 
The most comprehensive study into modality disruptiveness was performed by Arroyo, Selker \& Stouffs [1]. Their work investigated the disruptive properties of five notification modalities: heat, smell, sound, vibration and light. Disruption was measured subjectively by the participants. The authors reported that their results were not statistically significant, providing only anecdotal evidence for differences between notification modalities.

A later experiment by Arroyo \& Selker [2 that considered heat and light based notifications attempted to demonstrate that disruptiveness differs with modality. Subjects were asked to play a text-based adventure game with disruptiveness measured primarily by the number of mistakes made post-notification. The data reported by the authors did not reveal significant differences between the disruptiveness of the modalities, although the authors asserted that light was superior to heat in their conclusions.

Latorella [19] performed a study into the properties of multimodal notifications in aeroplane cockpits. Latorella reported that there were subtle differences between the visual and audio alerts, but that there were also interaction effects between the modalities of the task and the notification. Latorella concluded that the modality of the notification had an effect on response time, errors in the notification task, and errors in the primary task post-notification.

Arroyo, Selker \& Stouffs [1] suggested that previous exposure to a modality may reduce the negative effects of the notification. Other research has shown that training can reduce the disruptiveness of notifications 9, as can familiarity with the primary task [10] which may support their conclusion. This would suggest that common methods of notification (such as an audio beep) should be less disruptive than uncommon methods (such as an olfactory notification).

There is a conflict in some of the literature regarding the relationship between modality and disruptiveness, and the study by Arroyo, Selker \& Stouffs [1] did not provide knowledge useful to multimodal system developers. A comprehensive study is needed that will investigate how disruptive and effective notifications delivered in different modalities really are; this will help developers to include these modalities when developing multimodal systems.

\section{Design and Method}

An experiment with a within-subjects repeated-measures design was carried out in order to evaluate the disruptiveness and effectiveness of notifications in different modalities. Participants attempted to complete a primary task while notifications instructed the participant to carry out a brief secondary task. The primary task was a card-matching game and the secondary task was to receive a notification and acknowledge it by pressing one of three buttons. The notifications were presented in visual, audio, tactile and olfactory modalities. The independent variable was the modality of the notification and the dependent variables were the disruption in the primary task and the speed and accuracy of responses in the secondary task. The experiment was carried out with 27 participants (14 male and 13 female). The participants included 20 people in the 18-30 age group, 4 people aged 31-45 and 3 aged 46-60. 


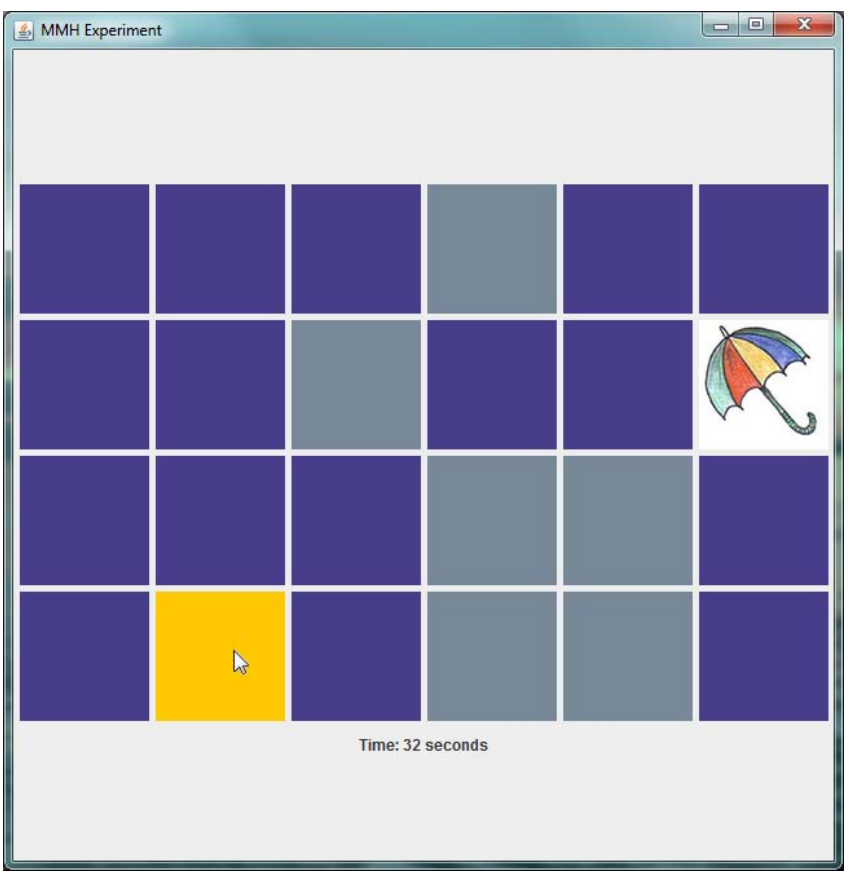

Fig. 1. A card-matching game in progress. The player has already matched 6 cards and is attempting to match the 'umbrella' card. The timer at the bottom of the screen shows the player has 32 seconds remaining.

\subsection{Primary Task}

A primary task was desired that encouraged the type of cognitive workload experienced by a person in their own home. This would provide a more realistic picture of how multimodal notifications might interfere with home life. McGeeLennon, Wolters \& McBryan 22] used a digit span test to evaluate serial recall, and Arroyo \& Selker [1] used a proof-reading test for their primary task. While these tasks have been repeatedly employed in experiments to build a mental workload, the type of workload they encourage is more likely to be encountered in an office environment than at home. A later experiment by Arroyo \& Selker [2] used a computer game, which was more likely to produce the desired type of cognitive workload; however their game was complex, time-consuming and difficult to interpret.

The task chosen was a simple card-matching game called 'Concentration' (also known as Memory or Pairs). In concentration, pairs of cards are presented face-down to the player. The player can then turn over two cards per turn in an attempt to find the pairs and remove them from the game (see Fig. 1). Psychologists have used this task in experiments to better understand how the brain processes information; in particular, the game has been used to evaluate how and why adults can outperform children despite the accepted view that children 

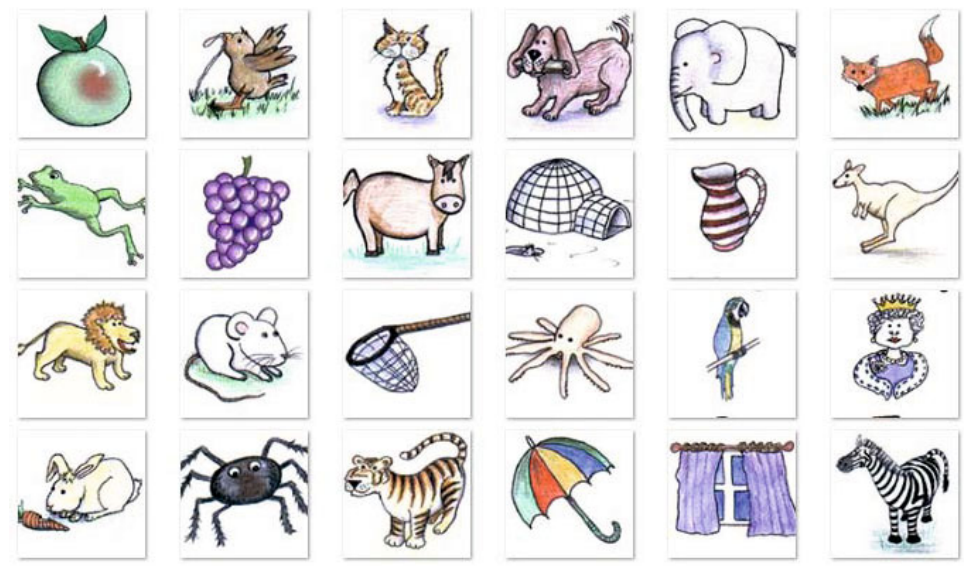

Fig. 2. Icons used in the primary task

have superior visual-spatial memory skills 41326]. Schumann-Hengsteler [26] suggests that the game of Concentration may not be entirely visual-spatial, arguing that adults have the ability to re-encode the information on the cards. A picture of a boat can be remembered by the picture itself, the verbal label 'boat', or simply by understanding what a boat is. In doing this, adults makes more efficient use of their memory which provides a greater performance benefit than the superior visual-spatial memory of a child player.

Simple icons were displayed on the cards to allow for the 're-encoding' phenomenon as described by Schumann-Hengsteler [26]. While there are various ISO standard pictogram sets, they are generally used for sign-making and most lack easily identified verbal labels. Instead, the game will used simple A-Z icons taken from an online speech therapy resource website1 as shown in Fig. 2.

A voluntary pilot study hosted on the project website 2 was run to evaluate the primary task and identify a suitable configuration. The experiment needed to be performed quickly enough that multiple trials and conditions could be completed, but there also had to be enough cards to ensure that the task was sufficiently challenging. The pilot consisted of 37 anonymous participants who each played three games of concentration with 16, 24 and 36 cards. The 24 card game provided an average completion time of 63.9 seconds, suggesting that a 24 card game with a 60 second time limit would allow many players to complete the game. This configuration was used in the experiment as shown in Fig. 1.

Concentration is a simple leisure activity that might well be carried out at home, it is a well-known game with very simple rules and it can quickly build a mental workload. The pilot study demonstrated that it was effective for the needs of the experiment and also allowed for the validation of the experimental measures as discussed in Sect. 3.4.

\footnotetext{
${ }^{1}$ Speech Teach UK, http://www.speechteach.co.uk

${ }^{2}$ The MultiMemoHome Project, http://MultiMemoHome.org
} 


\subsection{Secondary Task}

Gillie \& Broadbent 14 demonstrated that the complexity of the secondary task and its similarity to the primary task are the main factors in determining how disruptive a notification is. To isolate the effects of modality, the secondary task should be simple and as different from the primary task as possible.

The secondary task in our study required a participant to press one of three buttons in response to a notification. These buttons are large, physical, coloured buttons placed directly in front of the participant and fixed to the desk. Physically separate buttons encouraged the participants to look away from the screen and remove their hand from the mouse; this separation was intended to re-enforce the concept that participants must 'stop' the primary task to deal with the secondary one. Similar to the experiment carried out by McGee-Lennon, Wolters \& McBryan [22, the buttons were labelled with the terms "Heating", "Lights" and "Telephone" to provide home-related context as shown in Fig. 3. This was used to provide additional semantic links between the notifications and buttons where the delivery method could support it.
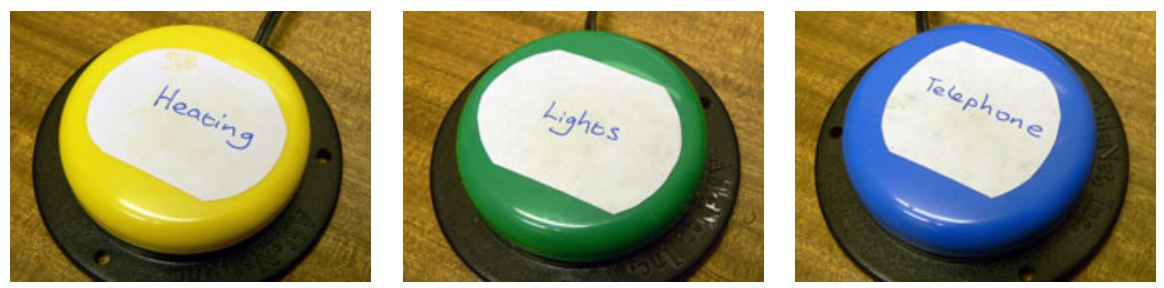

Fig. 3. Button colour \& labelling

\subsection{Notifications}

To evaluate the differences between notifications in different modalities, a wide range of unimodal notifications were designed for the experiments. These included common notification techniques such as text and speech along with less common notification modalities such as olfaction and abstract visual display. Eight unimodal notifications were developed for the experiment as shown in Table 1

The text and pictogram notifications were delivered directly into the game window to the top of the play area. No additional hardware was required for this configuration. The abstract visual display was created with a short-throw projector positioned to project a coloured light against the wall adjacent to the participant. The projector was deliberately aligned so that the projection lay in the peripheral vision of the participant, as shown in Fig. 4a,

In all the audio conditions the notifications were delivered through a pair of Sennheiser HD 25-1 II closed-back headphones as shown in Fig. 4b, These headphones helped to prevent background noise from causing interference.

${ }^{3}$ PacDV Sound Effects, http://www.pacdv.com/sounds/ 
Table 1. Modal Specification

\begin{tabular}{ccc}
\hline & \multicolumn{2}{c}{ Message } \\
\cline { 2 - 3 } Delivery Method Heating & Lights & Telephone Justification \\
\hline
\end{tabular}

\section{Visual}

Text

Pictogram

IEC-60878 IEC-60878 ISO-7001 Thermometer

Abstract Visual Yellow Green Light Light
"Heating" "Lights" "Phone" A simple one-word message displayed in a large bold font above the game.

1 Taken from two international stanLight Telephone dards; IEC-60878 and ISO-7001.

Light
Blue Light Projector used to shine a coloured light against the wall. The colour of the light matched the colour of the correct button.

\section{Auditory}

Voice

Earcon

Auditory Icon

Gas
Ignition

Light

Phone

Switch

Dialing Click (x2) Beeps

$\begin{array}{lll}\text { Spoken } & \text { Spoken } & \text { Spoken } \\ \text { "Heating" } & \text { "Lights" } & \text { "Phone" }\end{array}$

Marimba
Created using the same synthetic voice that was used by McGeeLennon, Wolters \& McBryan 22].

\section{Tactile}

Tacton

multiLP textLP voiceLP

Tactons varied in rhythm and were taken from an experiment by Brewster \& Brown [6].

\section{Olfactory}

Aromacon

Dale Air

Dale Air

Dale Air

Smells were selected based on in-

"Dark

"River-

"Rasp-

formation from an experiment by

Chocoberry" 


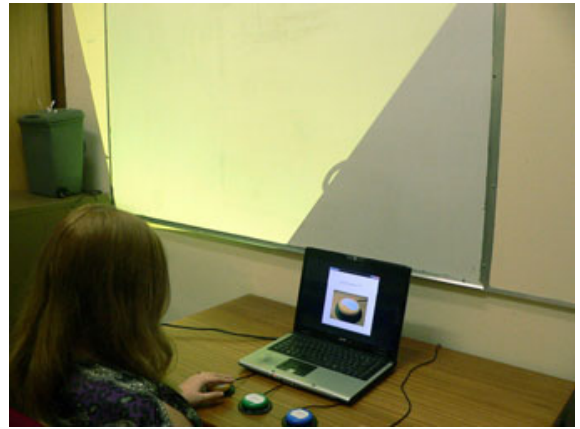

(a) Abstract Visual

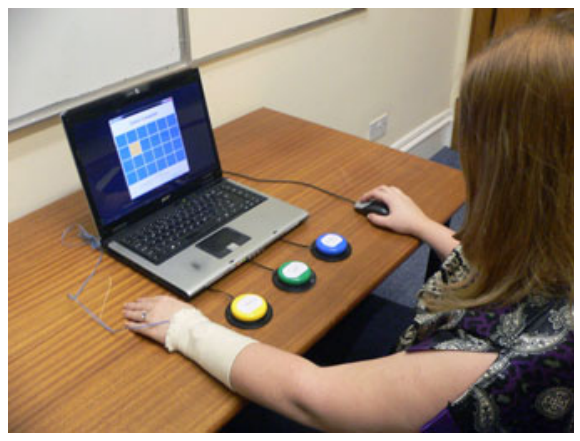

(c) Tactile

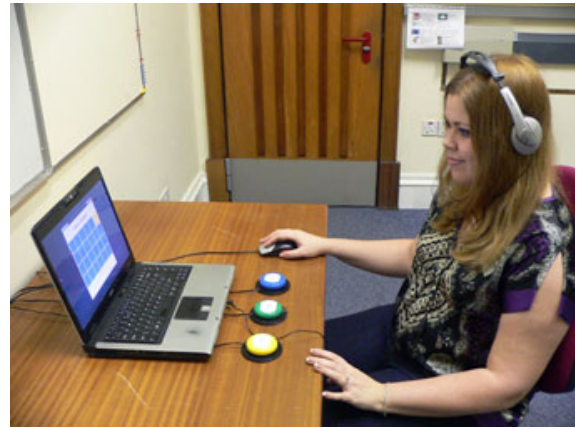

(b) Audio

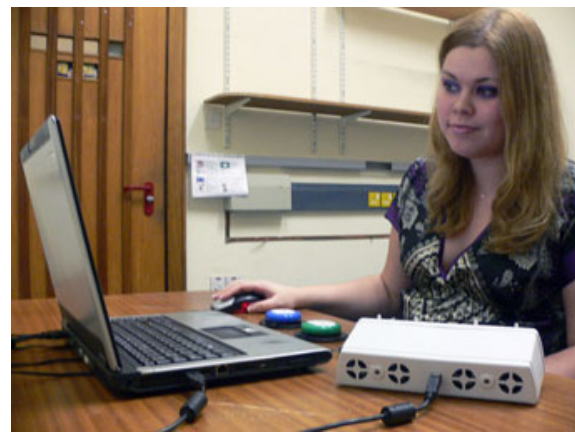

(d) Olfactory

Fig. 4. Experimental configurations

Tactile notifications were delivered via an Engineering Acoustics Inc. C2 vibrotactile actuator 4 powered by a small amplifier. This was secured to the top of the wrist on the participant's non-dominant hand with a stretchable bandage as shown in Fig. 4c in order to simulate notifications delivered from a watch. The device has a very low latency and was able to create precise tactile messages.

The olfactory notifications were delivered using a Dale Ain 5 Vortex Active smell device which has the capacity for 4 different smells. Scents are stored on 1-inch disks, which are blown by a fan to deliver the smell. Delivery times are much longer than the other devices (as the smells took longer to reach the nose), so to ensure the smells were delivered in a reasonable time frame the delivery device was placed directly in front of the participants as shown in Fig. 4d,

The notifications used in the study were all powered by off-the-shelf technology, so any commercial product providing interaction in these modalities would be likely to offer it at a similar quality. The notifications used in the experiment are defined in Table 1 which also shows how the unimodal notifications were grouped by sensory apparatus.

\footnotetext{
${ }^{4}$ Engineering Acoustics, http://www.eaiinfo.com

${ }^{5}$ Dale Air, http://www.daleair.com
} 


\subsection{Measurements}

Other experiments into disruptiveness have examined errors introduced into the primary task and change in activity rate post-notification 12 . Latorella [19] observed that activity rate and error rate disruptions have distinctly different properties.

Other experiments investigating the properties of concentration have used various techniques to measure performance. Schumann-Hengsteler [26] measured the number of missed opportunities, Baker-Ward \& Ornstein [4] measured the number of perfect matches, and Gellatly, Jones \& Best [13] observed player strategy.

Another measure was created by combining perfect matches and missed opportunities which we called superfluous views. This is a measure of how many decisions (i.e. cards clicked) were not productive. When a card was viewed, that card was marked as 'seen'. Every subsequent viewing of that card which failed to match it to another card was considered a superfluous view. A high number of superfluous views suggested that the participant found it difficult to remember which cards were where. This metric was expressed as superfluous views per turn to prevent it being influenced by player speed or game completion. The activity rate of the participants was measured as turns per second.

These measures of performance were tested as part of the pilot study described in Sect. 3.1. Superfluous views per turn was found to provide the most accurate, reliable and stable measure of error rate in the primary task, while turns per second was confirmed to be an adequate measure of activity rate.

Notification performance was measured by response accuracy and response time. Response accuracy simply checked that that the button pressed corresponded to the notification delivered. Response time was measured as the time between starting notification delivery and the participant pressing a button. This did not reflect the true response time as it is difficult to separate the delivery time from the processing time, particularly for olfactory notifications. Delivery time was considered to be an inherent trait of the modality, and as such separating it from the overall response time would not be representative of real-world performance.

\subsection{Hypotheses}

In order to explore the relationship between the effectiveness and disruptiveness of notifications and the modality used to deliver them, the following hypotheses were tested by the experiment.

H1: Error rate in the primary task will vary with the notification modality ( $m e a-$ sured as superfluous views per turn). Based on evidence provided by Arroyo, Selker \& Stouffs [1, it was hypothesised that people will be more disrupted by notifications that they do not encounter regularly. As olfactory notifications are very uncommon, it is likely they will prove to be the most disruptive.

H2: Activity rate in the primary task will vary with the notification modality (measured as turns per second). Other work has shown that introducing notifications has a significant impact on the speed at which the primary task is performed [17]. It is hypothesised that some modalities will have a more significant impact on the speed at which the primary task is performed than others. 
H3: The accuracy of notification responses will vary with notification modality (measured as the percentage of responses correct, incorrect and missed). It is hypothesised that inherent differences between the delivery methods will have a significant impact on how easy the notifications are to interpret.

H4: The speed at which participants respond to notifications will vary with the notification modality (measured as the average response time). Latorella [19] showed that audio notifications were responded to quicker than visual ones.

\subsection{Procedure}

At the beginning of each trial participants were given an information sheet and consent form, then asked to take a short demographic survey to collect gender and age information. Participants were also asked to self-assess their sensory ability on a 21-point Likert scale.

Each participant then completed a control condition (which had no notifications) and 4 experimental conditions. During the visual and audio conditions each participant would only see one of the delivery methods described in Sect. 3.3. Participants were counter balanced to ensure even coverage of the different delivery methods. With 27 participants in the visual condition, 9 received textual notifications, 9 received pictograms and 9 received abstract visual notifications (and similarly for audio). As the tactile and olfactory conditions both use a single delivery method the same configuration was used for all 27 participants. The control condition always came first, while the experimental conditions were delivered in a random order. As such the experimenter and participant were both blind to the order or type of experimental conditions.

Each experimental condition consisted of a training segment and five games (except the control condition, where training was not required). At the start of each condition a screen described the type of notification and the hardware was configured as required (see Sect. 3.3). Each participant was given the opportunity to make minor comfort adjustments (such as volume) before each condition began.

Participants were trained by introducing each notification in turn and associating them with the correct button. Notifications were then delivered randomly until the participant had correctly acknowledged 6 sequential notifications. The participant was informed and corrected if a mistake was made. This training helped to ensure that each participant had fully understood the links between the notifications and buttons at the start of each experimental condition.

With the training complete, the participant would then play five games of concentration. In each game 3 notifications were delivered at random points with buffers between them to prevent overlap. A large buffer at the end of the game helped to ensure that quick players could not finish before all the notifications had been delivered. At the end of every game the participant was provided with the opportunity to rest before the next game started.

Once all the conditions had been completed participants were paid and offered the opportunity to ask questions. The experiment required around 50 minutes per participant. 


\section{Results}

H1: Error rate in the primary task will vary with the notification modality (measured as superfluous views per turn).

Fig. 5 shows a comparison between error rate and the notification modality. A within-subjects one-way ANOVA was performed on superfluous views per turn for each modality, which showed significant differences between the means of the conditions $(F(4,101)=16.57, p<0.0001)$. Post hoc Tukey tests revealed that the significant differences were between the control and the experimental conditions; there were no significant differences in error rate between any of the conditions with notifications. The data did not support the hypothesis, suggesting that there is no connection between task disruption and the modality of the notification.

H2: Activity rate in the primary task will vary with the notification modality (measured as turns per second).

Fig. 6 shows the relationship between activity rate and notification modality. A within-subjects one-way ANOVA showed a significant effect of notification modality on the speed of the player $(F(4,101)=8.76, p<0.0001)$. Post hoc Tukey tests identified two groups: one containing the visual and audio conditions and the other containing tactile and olfactory. These two groups were significantly different from each other. Olfactory and tactile notifications introduced

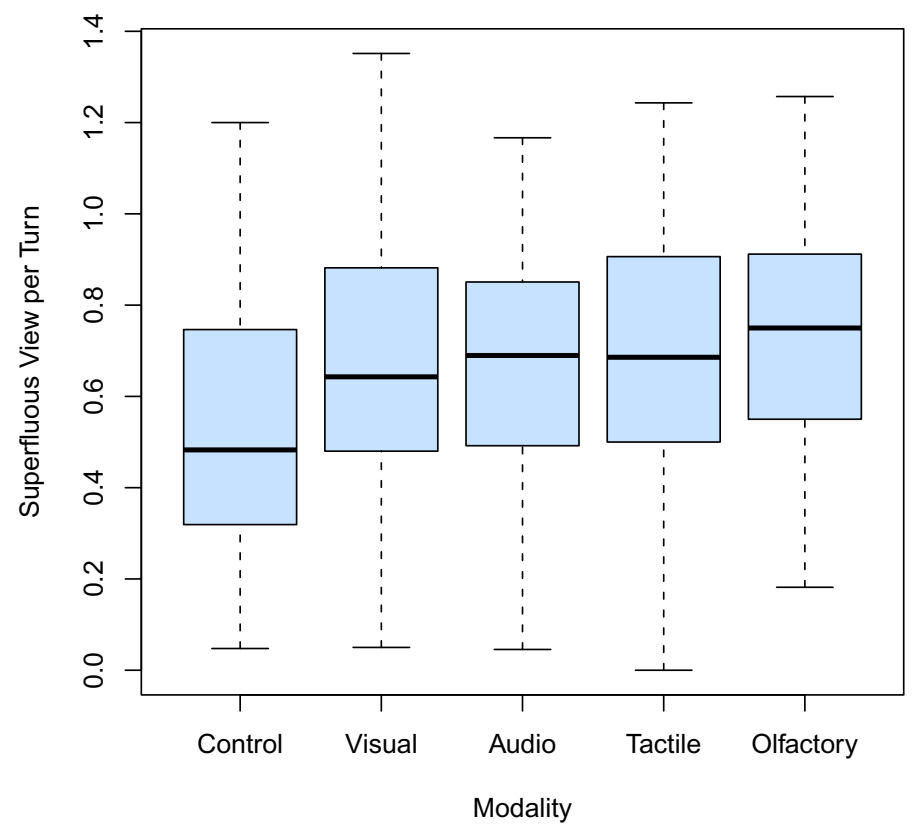

Fig. 5. Graph of Error Rate and notification modality. No notifications were delivered in the control condition. Superfluous views per turn has a theoretical range of $0-2$. 


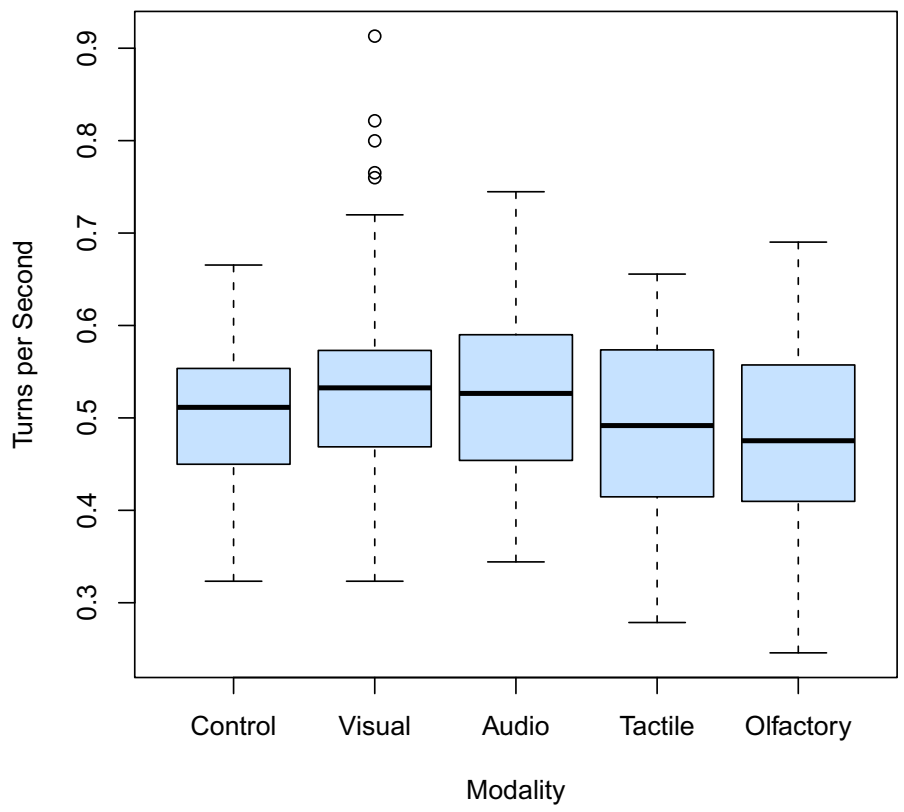

Fig. 6. Graph of turns per second and modality. No notifications were delivered in the control condition.

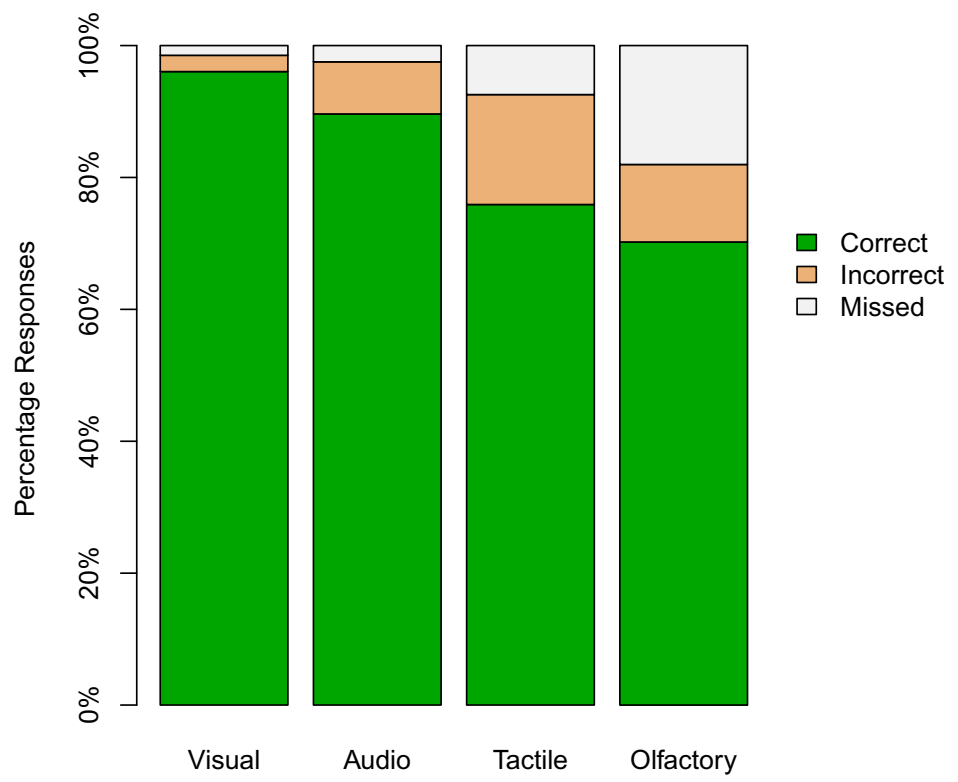

Fig. 7. Graph of responses by modality 


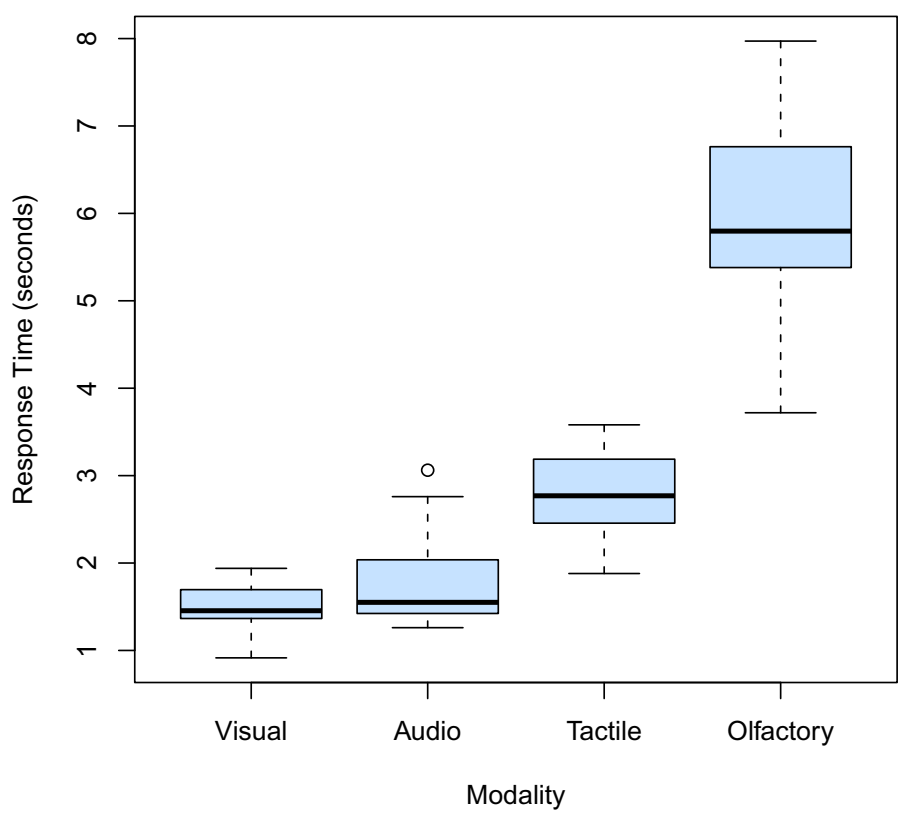

Fig. 8. Graph of response time and modality

a temporal disruption, slowing the players, while visual and audio conditions appeared to prompt the player to speed up resulting in a higher rate of activity. The evidence supports the original hypothesis that that notification modality will influence the rate of activity in the primary task.

H3: The accuracy of notification responses will vary with notification modality (measured as the percentage of responses correct, incorrect and missed).

The number of correct responses, as shown in Fig. 17, was evaluated with a KruskalWallis one-way ANOVA which found a significant effect of modality on correct responses $\left(\chi^{2}(3)=41.2, p<0.0001\right)$. The audio and visual modalities had a high correct acknowledgement rate with means of $90 \%$ and $96 \%$ respectively. Tactile and olfactory did not perform as well, scoring $76 \%$ and $70 \%$ respectively.

Modality was also found to have an effect on responses incorrect $\left(\chi^{2}(3)=\right.$ $24.96, p<0.0001)$ and missed $\left(\chi^{2}(3)=31.77, p<0.0001\right)$. The visual notifications had the lowest number of incorrect responses at $2.5 \%$, while the tactile condition had the highest at $16.7 \%$. More olfactory notifications were missed than any other modality. For visual and audio, very few of the notifications were missed. The evidence in this case strongly supports the hypothesis that there is an effect of modality on notification response accuracy.

H4: The speed at which participants respond to notifications will vary with the notification modality (measured as the average response time). 
This hypothesis was evaluated with a within-subjects one-way ANOVA over the average response time for each modality, which found a significant effect of modality on response time $(F(3,75)=322.09, p<0.0001)$. Average response time was calculated using the response times for both correct and incorrect acknowledgements and is shown in Fig. 8 .

\section{Discussion}

The study presented here show that the introduction of notifications was disruptive to the primary task, causing a significant jump in the error rate. However, there were no significant differences between the modalities, implying that the notifications were all equally disruptive. Activity rate was not significantly different from the control condition for any of the experimental conditions, despite the increased time demand on the participant. However, the visual and audio conditions did result in significantly higher activity rate than the tactile and olfactory conditions.

Arroyo, Selkers \& Stouffs [1 had tested disruption subjectively, but found no significant differences in any objective measures. This study agrees with their results, suggesting that there is no relationship between notification modality and error rate. Cellier \& Eyrolle [10] suggested an interrupted person takes a 'mental snapshot' of the task at hand when interrupted and then uses that information to resume the task later. The results of this experiment suggest that the modality of the notification does not affect the process of pausing and resuming a task and that as a result, the modality of the notification does not influence the error rate in the primary task.

Anecdotal evidence suggests that disruption could be greater for modalities which the user is unfamiliar with [12]; this is backed up by research showing that disruption effects can be reduced through experience or training 91423. This experiment did not measure familiarity, but the difference between visual and auditory activity rate and tactile and olfactory activity rate could be explained by this. Given the ubiquity of visual and audio devices and the relative scarcity of tactile and olfactory ones, it seems reasonable to assume that participants were more 'familiar' with visual and auditory notifications. This might provide an increased ability to intercept and process these notifications. However, further experiments would be needed to evaluate this.

The effectiveness of the notifications seems to be attributed to their individual properties. The olfactory notifications, which took the longest to deliver, showed a very long response time. Figure [7] shows olfactory and tactile notifications, which lacked semantic links to the buttons, had the lowest number of correct responses. Tactile notifications, which are targeted and attention-grabbing, had a lower 'miss' rate compared to olfactory notifications but were more likely than olfactory to be misinterpreted. This suggests that the individual properties of each modality are directly linked to their effectiveness as notifications.

The visual and auditory notifications performed well, as they were quick to deliver and provided a semantic link to the buttons (with the exception of the Earcons). The tactile notifications showed an unexpected 'response lag', as shown 
in Fig. 8. In addition, many of the participants verbally expressed that they had forgotten the button/notification associations at the start of the tactile condition, which did not occur with the other notifications. Hoggan \& Brewster 15] carried out a similar experiment but did not report any problems with tactile training or recall, suggesting that this issue could be the result of poor notification design. However, the tactile notifications had more correct responses than the olfactory ones, which could simply be the participants expressing a lack of confidence in their ability to correctly identify the notifications. This could also explain the increased response time.

The olfactory notifications could be said to have performed the poorest as they have the longest response time, the lowest number of correct responses and also caused a decrease in player speed compared to visual or audio notifications. Smell technology is rarely examined in multimodal research; smells are difficult to control, they linger after delivery and the technology relies on limited-lifespan chemical components. For these reasons it is often unfairly dismissed as completely impractical, yet in reality artificial smells are common in the home from air fresheners to perfumes and scented candles. This suggests that people might also be willing to accept smell-based technology in their home.

If a home care system was equipped with the same modalities used in this experiment, it would be able to adapt to a range of situations. For example, a reminder notification to lock doors may be considered non-urgent in the afternoon and be displayed textually. Once night falls it becomes more important, so audio notifications might be used instead to alert the target user. Another example would be an elderly person living alone who must take certain medicines daily. To remind the person to take their medicine, a message could be sent to their TV screen; however, if the reminder system detects a social situation, any potentially embarrassing notifications could be re-routed through a private tactile device or an ambient visual display. If this person also had a visual impairment then Auditory Icons or Earcons could be substituted for speech in the same situation.

The study presented here has shown that there is no 'best modality'; all of the unimodal notifications examined by the experiment could be effective notifications. We agree with other researchers [1 21 22 28 $]$ who have argued that a range of modalities should be used to provide more appropriate and effective interactions. Notification modalities should be chosen to suit the situation based on factors such as user preference, message sensitivity, social setting, message complexity, message urgency and user impairment.

\section{Conclusion and Future Work}

The study presented here has shown that there are a wider range of modalities available to home care developers than are commonly used. The results of the study will help developers to include these modalities, allowing them to create home care technology that is more appropriate, acceptable and effective. This knowledge will also help to expand upon models of interruptibility in the home, such as that developed by Vastenburg, Keyson and Ridder [27]. Their model considers message urgency and user engagement to evaluate notification 
acceptability, which is very much a step in the required direction. However, their model does not include modality or social acceptability, and it does not make provisions for sensory impairment. New models are required that will satisfy the needs of home care patients. In addition, more research is needed in order to fully understand the acceptability of different notification modalities when delivering messages of varying urgency or importance.

In conclusion, the results of this experiment show that the modality of the notification will not affect the primary task, but it is the notification itself which is disruptive. In addition, the results highlight how effective and practical different notification modalities can be. This will help to guide future development of multimodal technology by encouraging developers and researchers to take advantage of a wider range of modalities, allowing the adoption of techniques which are more effective, appropriate and practical.

Acknowledgments. This work was funded by the EPSRC (grant number EP/G069387/1). We would like to thank the anonymous reviewers for their feedback and insights, those who participated in the study and Miss M. Vernigor for modelling the experiment.

\section{References}

1. Arroyo, E., Selker, T., Stouffs, A.: Interruptions as multimodal outputs: which are the less disruptive? In: Proceedings of Fourth IEEE International Conference on Multimodal Interfaces, pp. 479-482 (2002)

2. Arroyo, E., Selker, T.: Self-adaptive multimodal-interruption interfaces. In: International Conference on Intelligent User Interfaces, pp. 6-11 (2003)

3. Bailey, B.P., Konstan, J.A., Carlis, J.V.: The effects of interruptions on task performance, annoyance, and anxiety in the user interface. In: Proceedings of INTERACT, pp. 593-601 (2001)

4. Baker-Ward, L., Ornstein, P.A.: Age differences in visual-spatial memory performance: do children really out-perform adults when playing Concentration? Bulletin of the Psychonomic Society 26(4), 331-332 (1988)

5. Berg, W.P., Alessio, H.M., Mills, E.M., Tong, C.: Circumstances and consequences of falls in independent community-dwelling older adults. Age and Ageing 26(4), 261-268 (1997)

6. Brewster, S., Brown, L.M.: Tactons: structured tactile messages for non-visual information display. In: ACM International Conference Proceeding Series, vol. 53, pp. 15-23 (2004)

7. Brewster, S., McGookin, D., Miller, C.: Olfoto: designing a smell-based interaction. In: Conference on Human Factors in Computing Systems, pp. 653-662. ACM, New York (2006)

8. Burmistrov, I., Leonova, A.: Do Interrupted Users Work Faster or Slower? The Micro-analysis of Computerized Text Editing Task. In: Proceedings of HCI International on Human-Computer Interaction: Theory and Practice, pp. 621-625 (2003)

9. Cades, D.M., Trafton, J.G., Boehm-Davis, D.: Mitigating disruptions: Can resuming an interrupted task be trained? In: Human Factors and Ergonomics Society Annual Meeting Proceedings. Human Factors and Ergonomics Society, vol. 50(3), pp. 368-371 (2006) 
10. Cellier, J.-M., Eyrolle, H.: Interference between switched tasks. Ergonomics 35(1), 25-36 (1992)

11. Cohen, S.: Aftereffects of stress on human performance and social behavior: A review of research and theory. Psychological Bulletin 88(1), 82-108 (1980)

12. Department of Health: National Service Framework for Older People (2007)

13. Gellatly, A., Jones, S., Best, A.: The Development of Skill at Concentration. Australian Journal of Psychology 40(1), 1-10 (1988)

14. Gillie, T., Broadbent, D.: What makes interruptions disruptive? A study of length, similarity, and complexity. Psychological Research 50(4), 243-250 (1989)

15. Hoggan, E., Brewster, S.: Designing audio and tactile crossmodal icons for mobile devices. In: Proceedings of the 9th International Conference on Multimodal Interfaces, pp. 162-169 (2007)

16. Hoggan, E., Crossan, A., Brewster, S., Kaaresoja, T.: Audio or tactile feedback: which modality when? In: Proceedings of the 27th International Conference on Human Factors in Computing Systems, pp. 2253-2256. ACM, Boston (2009)

17. Kapitsa, M., Blinnikova, I.: Task performance under influence of interruptions. In: Operator Functional State: the Assessment and Prediction of Human Performance Degradation in Complex Tasks, pp. 323-329. Ios Pr. Inc., Amsterdam (2003)

18. Kane, R.A.: Long-Term Care and a Good Quality of Life: Bringing Them Closer Together. Gerontologist 41(3), 293-304 (2001)

19. Latorella, K.A.: Effects of Modality on Interrupted Flight Deck Performance: Implications for Data Link. Technical report. NASA Langley Technical Report Server (1998)

20. Mark, G., Gudith, D., Klocke, U.: The cost of interrupted work: more speed and stress. In: Proceedings of the Twenty-Sixth Annual SIGCHI Conference on Human Factors in Computing Systems, pp. 107-110 (2008)

21. McBryan, T., Gray, P.: A Model-Based Approach to Supporting Configuration in Ubiquitous Systems. In: Proceedings of Int. Conf. on Design, Specification and Verification of Interactive Systems, pp. 167-180 (2008)

22. McGee-Lennon, M.R., Wolters, M., McBryan, T.: Audio Reminders in the Home Environment. In: Proceedings of the 13th International Conference on Auditory Display, pp. 437-444 (2007)

23. Miyata, Y., Norman, D.: Psychological issues in support of multiple activities. In: User Centered Systems Design: New Perspectives on Human-Computer Interaction, ch.13, pp. 265-284. L. Erlbaum Associates Inc., Mahwah (1986)

24. Monk, C.A., Boehm-Davis, D.A., Trafton, J.G.: Very brief interruptions result in resumption cost. In: Proceedings of the Twenty-Sixth Annual Conference of the Cognitive Science Society, p. 1606 (2004)

25. Office for National Statistics: National Population Projections (2008)

26. Schumann-Hengsteler, R.: Children's and adults' visuospatial memory: The game concentration. Journal of Genetic Psychology 157(1), 77-92 (1996)

27. Vastenburg, M.H., Keyson, D.V., Ridder, H.: Considerate home notification systems: A user study of acceptability of notifications in a living-room laboratory. International Journal of Human-Computer Studies 67(9), 814-826 (2009)

28. Warnock, D.: A Subjective Evaluation of Multimodal Notifications. In: To Appear in Proceedings of Pervasive Health 2011, Dublin, Ireland (May 2011) 